\title{
Numerical simulations of an intake-compressor system
}

\section{Original article}

Article history:

Accepted: 16 July 2018

Published: 3 October 2018

This paper is the updated version of a paper originally presented at the GPPS Montreal 18 Conference, in Montreal, May 7-9 2018.

\section{Check for updates}

\section{*Correspondence:}

TK: thomas.kaechele@unibw.de

\section{Peer review:}

Single blind

\section{Copyright:}

() 2018 Kächele et al. @) This is an open access article distributed under the Creative Commons Attribution Non Commercial No Derivatives License (CC BY-NC-ND 4.0). Unrestricted use, distribution, and reproduction of the original work are permitted for noncommercial purposes only, provided it is properly cited and its authors credited. No derivative of this work may be distributed

\section{Keywords:}

CFD; inflow distortion; serpentine intakes; component interaction

\section{Citation:}

Kächele T., Rademakers R. P. M., Schneider T., and Niehuis R. (2018). Numerical simulations of an intake-compressor system. Journal of the Global Power and Propulsion Society. 2: 442-452. https://doi.org/10.22261/JGPPS.VQB6CK

\author{
Thomas Kächele ${ }^{1,{ }^{*}}$, Rudolf P. M. Rademakers ${ }^{1}$, Tim Schneider ${ }^{2}$, Reinhard Niehuis ${ }^{1}$ \\ ${ }^{1}$ Bundeswehr University Munich, Werner-Heisenberg-Weg 39, Neubiberg 85577, Germany \\ ${ }^{2}$ MTU Aero Engines AG, Dachauer Straße 665, München 80995, Germany
}

\begin{abstract}
Integrated propulsion plays a major role in future civil and military aircraft design. A key component of these systems are highly bent intake geometries. As the flow passes through such ducts, combined total pressure and swirl distortions are generated which have a negative impact on compressor performance, safety margin, and durability. Due to weight and space limitations, a close coupling of intake and compressor is necessary. An experimental test case including a highly bent intake geometry and a state of the art turbofan engine was established and extensive measurement data was acquired. This publication compares results of three different numerical approaches to this test data: Isolated intake simulations, isolated compressor simulations with distorted inflow conditions, and a coupled simulation of intake and three stage compressor.

The isolated intake simulation is able to reproduce the static wall pressure field of the intake as well as the occurring flow separation. Towards the interface plane to the compressor however, significant deviations are observed. The upstream effect of the compressor working under the combined pressure swirl distortion is assessed via the second simulation approach. The influence of the swirl and total pressure distortion on the compressor is first simulated separately and then compared to the impact of the combined distortion. The coupled intake-compressor simulation reveals the manipulation of the intake flow field by an upstream static pressure field. In contrast to experiments a slightly unsteady operation point and an asymmetric intake flow field were observed.
\end{abstract}

\section{Introduction}

Modern civil and military aircraft concepts often feature short and highly contoured engine intake systems. The reduction of overall aircraft drag lies in the focus of the civil boundary layer ingesting (BLI) aircraft concept, while the hiding of the highly reflective fan plane from a direct line of sight motivates military applications. As the flow passes through these ducts, secondary flows arise due to surface curvature and changes in cross-sectional area and shape. Towards the Aerodynamic Interface Plane (AIP) between intake and compressor, total pressure and swirl distortion arise. The subsequent compressor working under these inhomogeneous inflow conditions experiences losses in efficiency and performance. A reduced stall margin leads to the risk of engine surge and severe damage of the engine.

In the usual design process, the intake geometry is designed in an isolated approach using numerical simulations and wind tunnel experiments. The assessed AIP flow distortion is then quantified by distortion parameters, which are compared to safety limits specified for the subsequent engine.

A compressor working under non-uniform inlet conditions shows complex flow physics (Longley and Greitzer, 1992). Three types of inflow distortions exist: total temperature, total pressure, and swirl 
distortion. In the case of bent intake ducts, only the latter two are of relevance. An incoming swirl distortion changes the incidence angle of the compressor blades which directly impacts the local blade work input in terms of total pressure and total temperature rise. Severe incidence angles may provoke stall and engine surge. An incoming total pressure distortion is always related to a distortion in axial velocity in the distorted sector. It changes the $p_{t, \mathrm{AIP}} / p_{\text {out }}$ characteristic of the compressor and thus the $p_{\mathrm{AIP}} / p_{\text {out }}$ characteristic, leading to a reduced static pressure in front of the compressor which propagates upstream (Mazzawy, 1977; Longley and Greitzer, 1992; Lesser and Niehuis, 2014). Due to this induced pressure field, a mass flow redistribution takes place generating an additional swirl distortion upstream of the compressor. In a close coupled intake-compressor system, the intake secondary flow is affected by these additional pressure gradients. Investigations by Ma et al. (2018) indicate that the presence of a compressor can lead to an earlier reattachment of an upstream flow separation. Downstream of a rotor exposed to non-uniform inflow, a highly complex flow field occurs: The total pressure distortion which is convected through the fan is superposed by an additional total pressure and total temperature distortion due to the non-uniform work input. In the following stator, losses through corner separations can arise. As a consequence, the next stages and thus the whole compressor works under off-design conditions leading to changes in $m_{\text {red }} / \Pi_{t}$ characteristic, which can negatively affect the stage matching (Cumpsty, 2010).

In recent years, full annulus URANS simulations of (multi-stage) compressors working under distorted inflow allow a detailed investigation of the highly three-dimensional character of the flow field (Yao et al., 2010a,b). First, simple generic distortion patterns were investigated and compared to experimental data (Gunn et al., 2013; Lesser and Niehuis, 2014). Later also BLI typical distortion patterns have been investigated which feature both radial and circumferential total pressure profiles (Gunn and Hall, 2014). Weston et al. (2015) investigated the transport of the inflow distortion through a three stage fan, while recently, Page et al. (2017) focused on complex combined pressure swirl distortions like they also occur in bent intake ducts.

Within the scope of a research project, an experimental full size intake-engine test case was set up at the Bundeswehr University Munich. Experimental data was collected allowing detailed insight into the intake flow field and the AIP flow conditions. In the present publication, the abilities of three different numerical approaches in reproducing the measured flow field are evaluated: An isolated intake simulation, isolated full annulus low pressure compressor (LPC) simulations exposed to the measured AIP flow field, and finally a coupled approach of intake and LPC.

\section{Experimental test case}

In cooperation with MTU Aero Engines AG, the Institute of Jet Propulsion set up an experimental test case combining a generic highly contoured intake geometry and a state of the art MexJET turbofan engine (Rademakers et al., 2016). A schematic sketch of the intake and the three stage low pressure compressor section of the setup is given in Figure 1.

Prior to entering the intake at the duct inlet plane (DIP), the flow is guided through an airmeter, which is not shown in the drawing for reasons of clarity. A traversable measurement rake equipped with five-hole and pitot probes is part of the measurement equipment. It can be installed at the DIP to measure the inflow, needed as inlet boundary condition for CFD, or at the AIP to measure the duct flow distortion. In both cases, static and total pressures as well as the flow angles are measured at 120 positions within the respective plane. Due to the limited calibration range of the five-hole probes, flow angles larger than $\pm 30^{\circ}$ cannot be measured. Close to the wall, the total pressure is captured by three pitot probes at 24 circumferential positions. The inflow conditions are additionally measured by two boundary layer probes at $\phi=35^{\circ}$ and $\phi=215^{\circ}$. Along the duct surface, the static wall pressure is collected at 149 positions: along the symmetry line on the upper $\left(\phi=0^{\circ}\right)$ and lower $\left(\phi=180^{\circ}\right)$ side of the intake as well as five cross sections (cs-1 to $\left.c s-5\right)$. In total, the flow regime at eight different operation points along the operation line was measured. A detailed summary of the measurement campaign can be found in Rademakers et al. (2018).

The intake flow field lies in the focus of the present numerical investigations. The measurement equipment within the LPC only consists of total temperature as well as static and total pressure probes at four circumferential positions at the LPC outlet. This allows the assessment of the operation point $\left(m_{\text {red }} / \Pi_{t}\right)$, but no detailed flow measurements in flow cross sections between the blade rows as usually measured in compressor test rigs were performed so far.

\section{CFD setup}

The flow solver TRACE (Nürnberger, 2004; Kügeler, 2005) is used for the presented CFD simulations. This 3D finite volume code is developed at DLR-AT and was recently validated for full annulus calculations of low 


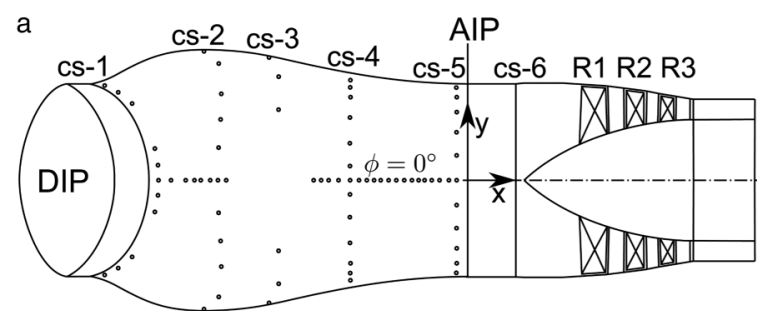

top view

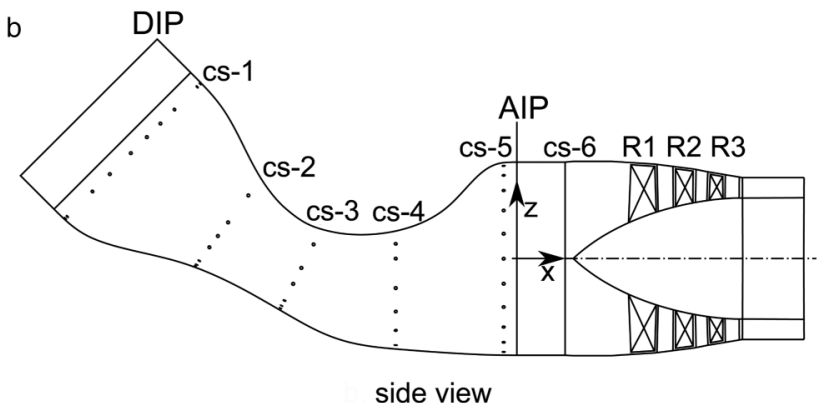

side view

Figure 1. Coupled intake-compressor setup with measurement positions.

pressure compressors working under distorted inflow conditions (Lesser and Niehuis, 2014; Schoenweitz and Schnell, 2016). It solves the discretized (U)RANS equations on structured multi-block meshes. Fully turbulent mode was used due to Reynolds numbers of $5.25 \cdot 10^{6}$ and $2.52 \cdot 10^{6}$ based on intake diameter and fan blade chord respectively.

Due to the lack of a best practice setup for bent intake ducts an extensive numerical parameter study including a mesh density study was carried out (Kächele et al., 2018). It showed the superiority of the streamline curvature correction proposed by Kožulović (Kožulović and Röber, 2006) for the $k-\omega$ turbulence model within the duct domain.

Within the compressor domain, turbulence is modelled by the Wilcox $k-\omega$ model with an additional correction for rotational effects proposed by Bardina and a stagnation point anomaly fix according to Kato-Launder (Kožulović et al., 2004). Applied on a full annulus simulation of all three LPC stages, the standard mesh density, which is used for the calculation of speed lines and calibrated by rig experiments, would exceed the limited resources available in industrial applications. Thus, a coarsening of the temporal and spatial resolution of the simulation was necessary. These efforts towards a setup, which is still able to reproduce the dominant flow features of the intake-compressor interaction, are summarized by Kächele et al. (2016). A smart coarsening of the blade rows downstream of Rotor 1 leads to a reduction of overall cell count by $70 \%$ to 100 million cells with a similar reproduction of the speed line in the operational point of interest. A relatively coarse time step of 36 outer time steps per fan blade passage was still able to reproduce the desired rotor-stator interactions. In coupled performance runs the calculation time for one rotor revolution on the available $128 \mathrm{CPU}$ was reduced to less than 2 days. This is necessary to account for the long expected transient oscillation time of the low frequent duct flow field of 12 to 15 rotor revolutions.

\section{Isolated intake simulation}

Isolated pre-test investigations (Kächele et al., 2018) indicated a weak unsteady behavior of the intake flow field. Its temporal average is thereby similar to the result of a steady simulation. Hence, no URANS computations are required for the assessment of the main isolated intake flow features such as the vortex system and the AIP flow field.

\section{Inflow boundary condition}

The measured DIP flow field shown in Figure 2 is used as inlet boundary condition. The local region of low total pressure close to the top of the DIP is the wake of a total temperature probe mounted shorty upstream. A swirl pattern with flow angles up to $15^{\circ}$ arises due to the unconventional aerodynamics of the engine test facility (Muth et al., 2009). Flow angles measured by the five-hole probes at the outermost radial position are kept constant towards the outer DIP wall. Constant values for total temperature and turbulence level of $1.3 \%$ are taken from measurements. The necessary turbulent length scale is set to achieve a nearly constant turbulence level within the contracting part of the intake.

\section{Comparison of CFD and experiment}

The desired mass flow rate is set by adjusting the constant static pressure ("Duct OD outlet" in Figure 3) at the outlet boundary condition $1.5 \cdot D_{\text {AIP }}$ downstream of the AIP until the wall pressure level at cross section $c s-1$ (cf. Figure 1) is matched. For reasons of clarity, subsequent comparisons comprise one single operational point 


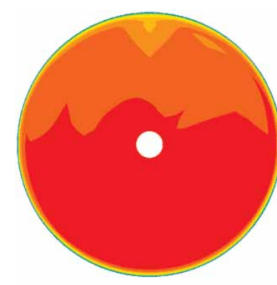

$p_{t, r e l}[-]$



0.90 .930 .960 .99

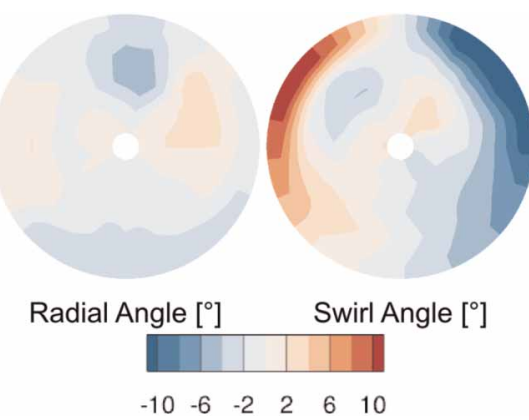

Figure 2. Measured flow conditions at duct inlet plane (DIP).

(corrected LPC shaft speed (Nlc) of 85\%). For higher or lower inlet mass flow rates, experimental as well as CFD data show similar wall pressure distributions and AIP patterns only scaled to higher/lower min values.

The intake flow field is determined by streamwise and cross flow pressure gradients that arise through centerline curvature and changes of cross-sectional area. In order to illustrate the relation between geometry and pressure gradients, the duct contour is added in the plot of the static wall pressures along the symmetry line in Figure 3.

Within the first half of the duct until $c s-3$ the cross-sectional area is reduced to $75 \%$ of the inlet area $A_{\text {DIP }}$, which leads to an acceleration of the flow. In parallel, the streamlines follow the bent duct contour leading to an additional pressure gradient. The pressure drop on the upper side $\left(\phi=0^{\circ}\right)$ is therefore larger than on the lower duct wall $\left(\phi=180^{\circ}\right)$. In this part of the intake, CFD results nearly perfectly align with experiments.

Both geometric trends reverse in the second half of the duct: cross-sectional area increases, while the duct surface on the upper side experiences a strong curvature in positive $z$-direction. These two changes in geometry lead to a significant adverse streamwise pressure gradient at $\phi=0^{\circ}$ on the upper side of the duct. At about $x / D_{\text {AIP }}=-0.55$ the low momentum boundary layer fluid is no longer able to follow the upper duct surface and separates (indicated as a pressure plateau). Note, that this plateau is formed slightly earlier in the experiments as predicted by CFD. Along with it, the static pressure level of this plateau is predicted higher by about 3.5\%. Both deviations are often reported for RANS computations of bent intake ducts (Tormalm, 2006; Delot and Scharnhorst, 2013; Kächele et al., 2018). While the pressure plateau in the numerical data stretches throughout the AIP, a slight pressure increase is observed in experiments. A good representation is also found for the crossflow pressure distribution of the isolated intake simulation slightly upstream of the flow separation within cross section $c s-4$, while a significant deviation is observed in $c s-5$ (cf. Figure 4). Along with the discussed three dimensional pressure gradient, centrifugal forces due to streamline curvature act on the fluid particles passing through the duct and give rise to secondary flows.

The AIP total pressure distortion given in Figure 5 is mainly a result of the accumulation of low momentum boundary layer material due to secondary flow and additional losses caused by the flow separation. In the measured data, this distortion is accompanied by a static pressure distortion at the same position. This compressor induced static pressure field leads to a mass flow redistribution visible as radial and circumferential flow angles towards the center of the static pressure distortion. The constant static pressure outlet condition $1.5 \cdot D_{\text {AIP }}$ downstream of the AIP in the isolated intake simulation does not represent this behavior. Hence, a larger circumferential extent of the distortion is predicted while the minimum total pressure is higher ( 0.85 vs. 0.8$)$. In contrast to the experimental data, CFD predicts a region of backflow in the upper half of the distortion. Thus, a particular swirl pattern arises in consequence of these low and negative axial velocities (Swirl angle $\theta=\operatorname{atan}\left(v_{\theta} / v_{x}\right)$, Radial angle $\alpha_{R}=\operatorname{atan}\left(v_{r} / v_{x}\right)$ ).

Even though the total pressure field is only slightly asymmetric, the region of backflow and hence the swirl distortion shows a significant asymmetry. In case of a homogeneous axial inlet boundary condition with constant flow variables, the AIP distortion pattern is $100 \%$ symmetric (cf. (Kächele et al., 2018)). It can thus be concluded that the intake flow simulation is very sensitive to asymmetries.

The measured AIP static pressure field can also be used as a boundary condition for the isolated duct domain ("Duct 2D outlet"). For such computations, the outlet boundary condition has to be placed within the AIP which reduces the possible extension of the flow separation. Again, the mass flow was set by adjusting the static pressure in $c s-1$. Using the original measured pressure field leads to an over prediction of the mass flow. Hence, the outlet profile was scaled to a higher pressure level by 5.2\%. The resulting AIP flow field (cf. Figure 5) shows significant improvements concerning the flow angle distribution. The total pressure distribution, however, is reduced both in size and intensity. 


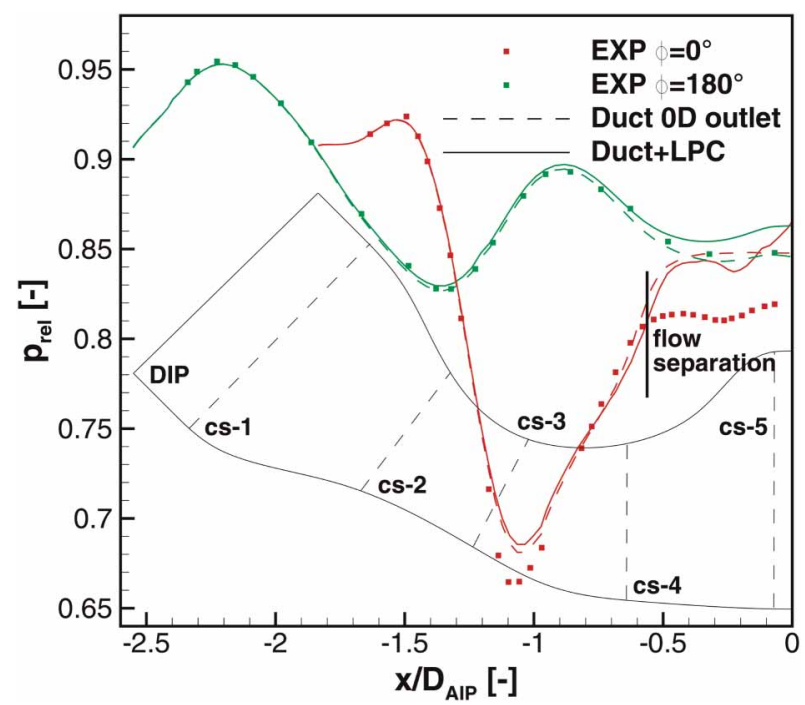

Figure 3. Comparison of experimental and CFD static wall pressure data along the centerline for the isolated and coupled intake simulation.

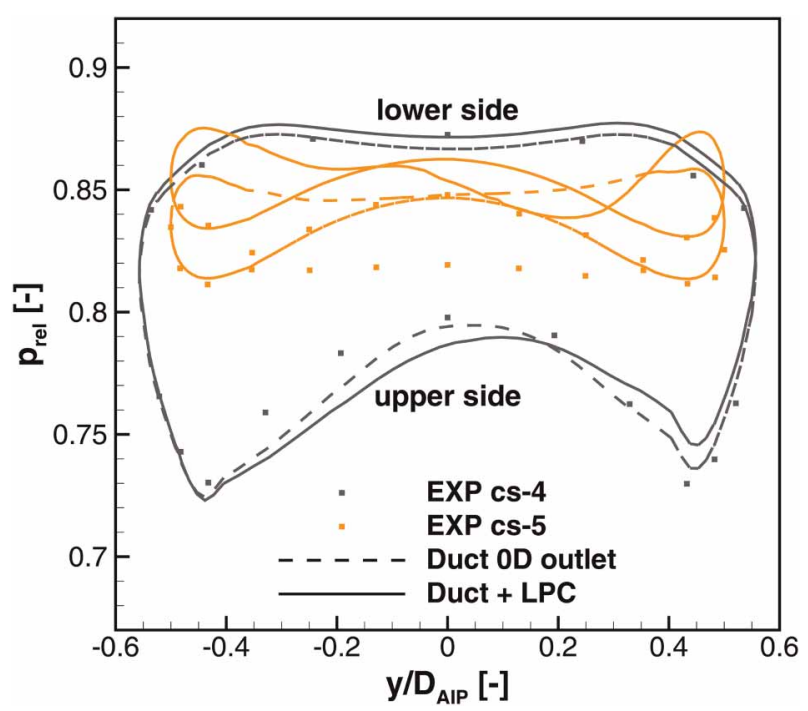

Figure 4. Comparison of experimental and CFD static wall pressures at cross-sectional planes cs-4 and $\mathrm{Cs}-5$.

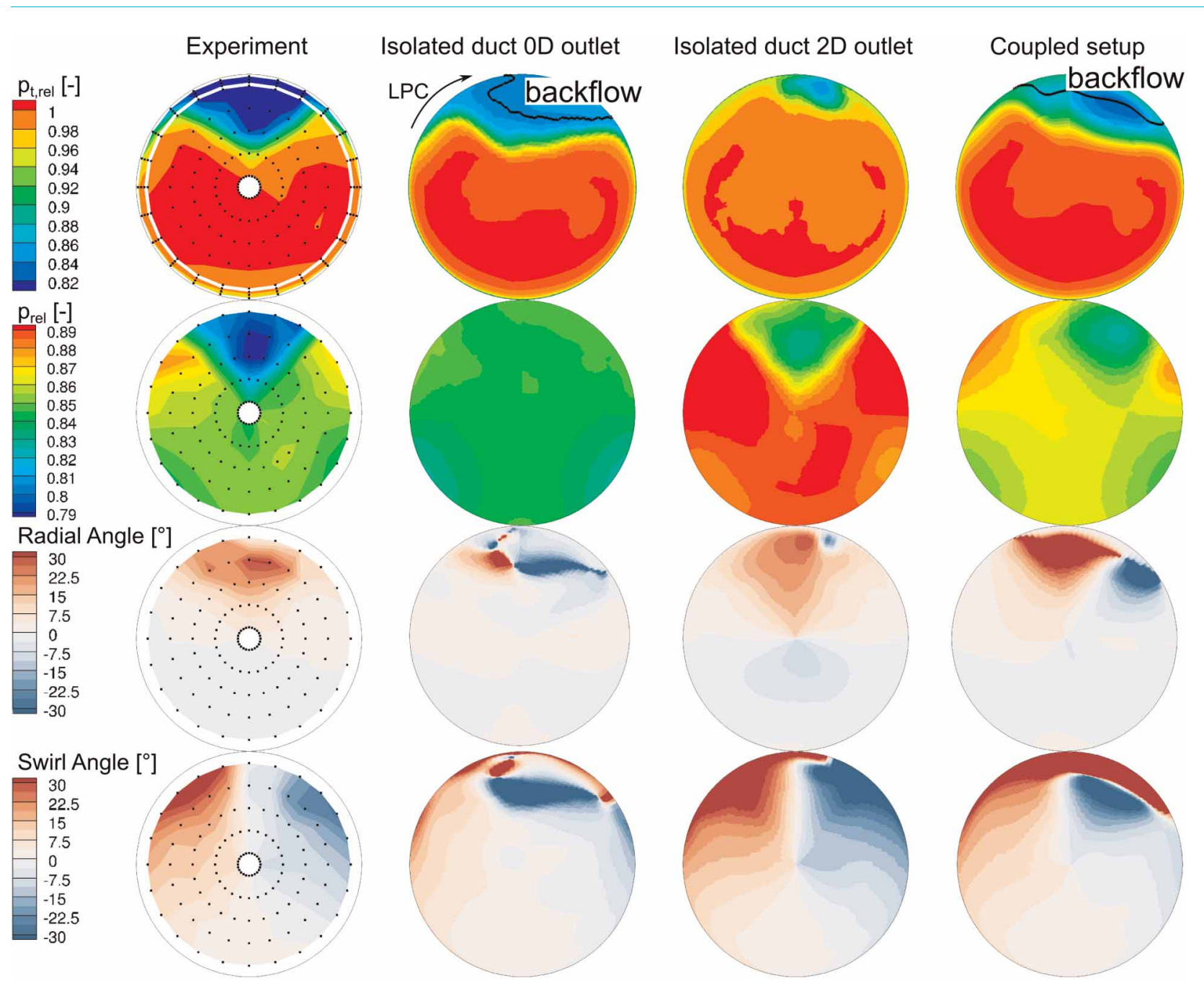

Figure 5. Comparison of experimental AIP flow field with results from the isolated intake simulation with constant outlet pressure (OD outlet), two dimensional outlet pressure (2D outlet) and the coupled simulation (Coupled setup). 


\section{Isolated compressor simulation}

In order to assess the reaction of the MexJET LPC on the AIP flow distortion without an interaction with the intake, full annulus URANS simulations of the isolated LPC domain downstream of the AIP (cf. Figure 1) are carried out first. These calculations use the measured AIP flow distortion (cf. Figure 5) as an inflow boundary condition. In this numerical approach, the total pressure and swirl component of the intake AIP distortion can be assessed separately to understand the isolated influence of the specific distortion component on the compressor. The three following patterns were applied:

1. swirl distortion

2. total pressure distortion

3. combined pressure-swirl distortion

The URANS simulations with distorted inflow require a convergence time for transient oscillation of about seven rotor revolutions. All presented simulations feature the same Nlc speed, AIP total temperature $T_{t, \text { AIP }}$ and outlet static pressure $p_{\text {out }}$. A reference simulation with a homogeneous, clean inflow is set up with a constant AIP total pressure of $p_{t, \text { ref. }}$. Hence, the LPC in both cases with total pressure distortions (case 2 and 3) operates at a $p_{t, \mathrm{AIP}} / p_{\text {out }}$ ratio with is reduced (throttled) by $3.3 \%$ compared to the clean reference case. The respective operation points (OPs) are determined from the temporal average over one rotor revolution and summarized in the compressor map in Figure 6. The presented speed line was calculated by steady passage simulations of the LPC and is added as an orientation. A slight difference between the clean reference OP and the speed line is due to the different simulation approach.

\section{Swirl distortion}

Comparing the OP of case 1 with the clean reference case reveals an increase in $m_{\text {red }}$ by about $1.1 \%$. Such an increase in $m_{\text {red }}$ was reported for a negative (counter-rotating) bulk swirl (Sheoran et al., 2012). The measured AIP flow angle distribution, however, indicates a strong motion towards the center of the low static pressure region in the AIP but no bulk swirl. In order to visualize the further development of the swirl distortion, the flow field in $c s-6$, shortly upstream of the spinner (cf. Figure 1) is depicted in Figure 7. In this plane, the radial component of the flow angle distortion is nearly annihilated $\left(\alpha_{R, \max }<3,5^{\circ}\right)$ and the swirl distortion is significantly decreased from $\theta_{\max }=30^{\circ}$ to $20^{\circ}$ compared to the AIP. Still, the particular swirl pattern remains unchanged with a slightly stronger co-rotating component. The detailed mechanisms between this swirl pattern and changes of local mass flow density are not fully understood at the moment and will be subject of further research. Concerning the total pressure ratio $\Pi_{t}$ of the compressor, the effects of the co and counter-rotating swirl components seem to cancel out each other, the $\Pi_{t}$ remains practically unchanged.

\section{Total pressure distortion}

Regarding the OP of case 2, an increase in $\Pi_{t}$ in combination with a decrease of $m_{\text {red }}$ is observed. This is comparable to a throttling of the LPC (movement along the speed line towards a higher $\Pi_{t}$ ). Again, the flow field in



Figure 6. Normalized compressor map including the $85 \%$ Nlc speed line and the operation points of the compressor simulations.



Figure 7. Flow field in cs-6 for isolated swirl distortion (case 1) contour levels according to Figure 5. 


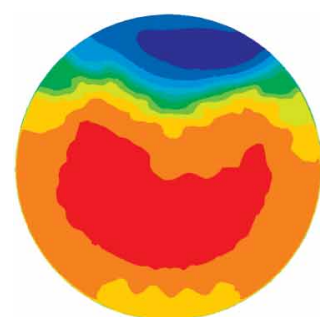

Total Pressure

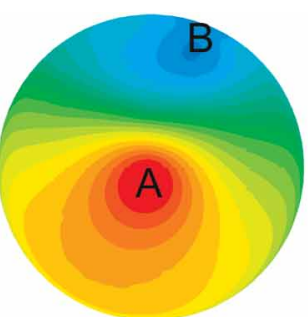

Static Pressure

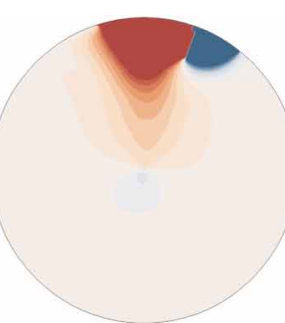

Radial Angle $\left[{ }^{\circ}\right]$

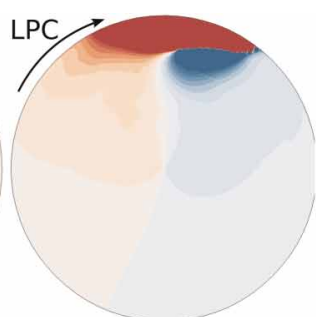

Swirl Angle $\left[^{\circ}\right]$

Figure 8. Flow field in cs-6 for isolated total pressure distortion (case 2) contour levels according to Figure 5.

cs- 6 is presented in Figure 8 to reveal the flow distortion close to the fan. In this case, the upstream influence of the fan can be noticed by an inhomogeneous static pressure field, which shows two phenomena: a region of high pressure in the center of the plane (denoted with "A") indicating the spinner stagnation point, and a pressure deficit ("B") close to the wall as a response of the LPC on the total pressure distortion. The resulting pressure gradient again leads to a compensating flow towards the center of this low pressure region. Due to the severeness of the total pressure distortion a very low axial velocity is predicted in this region, leading to in high radial and circumferential flow angles. Thus, the LPC already works under a combined pressure-swirl distortion, which additionally influences the fan rotor work input (Lesser and Niehuis, 2014). High swirl angles are observed downstream of the first rotor (not shown here for comprehensive reasons) leading to additional losses in the subsequent stator due to blade hub flow separation. While this swirl distortion is attenuated by the first stator row for the most part, the whole compressor works under a total pressure distortion as also described by Weston et al. (2015). Compared to the measured static pressure field as shown in Figure 5, the induced pressure deficit is under predicted in the simulations. Additionally, the slight turning in positive rotor (clockwise) direction by $\phi \approx 25^{\circ}$ is not seen to that extent in the experimental data.

\section{Combined swirl-pressure distortion}

The OP of case 3 resembles a superposition of both previous cases: a shift along the speed line in combination with an increased $m_{\text {red }}$. The additional increase in $m_{\text {red }}$, however, adds up to $0.7 \%$ while the pressure rise is reduced by about $1 \%$ compared to case 2 . In $c s-6$ the area of low total pressure in Figure 9 is reduced in size compared to case 2 and the static pressure field is less pronounced. This might be caused by the additional swirl distortion. The flow angle pattern in front of the spinner is a superposition of cases 1 and 2: The swirl distortion (case 1) is increased by an additional mass flow movement due to the induced static pressure field. Hence, the LPC in case 3 is exposed to a decrease in total pressure distortion but a strongly increased swirl distortion, which

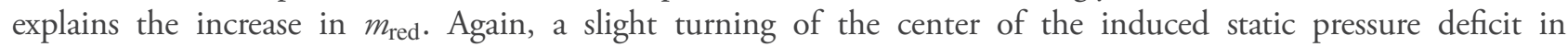
co-rotating direction by about $25^{\circ}$ is observed.

In the presented calculations, an upstream static pressure field is generated but limited in its upstream propagation by the AIP inlet boundary condition $0.6 \cdot D_{\text {AIP }}$ from the fan. With the use of a coupled approach, this pressure field, however, can interact with the intake flow field.

\section{Coupled intake-compressor simulation}

Due to the large simulation domain $\left(L \approx 4 \cdot D_{\text {AIP }}\right)$ and a complicated initialization procedure, a long calculation time for transient oscillation of more than 15 rotor revolutions was required for coupled intake-compressor
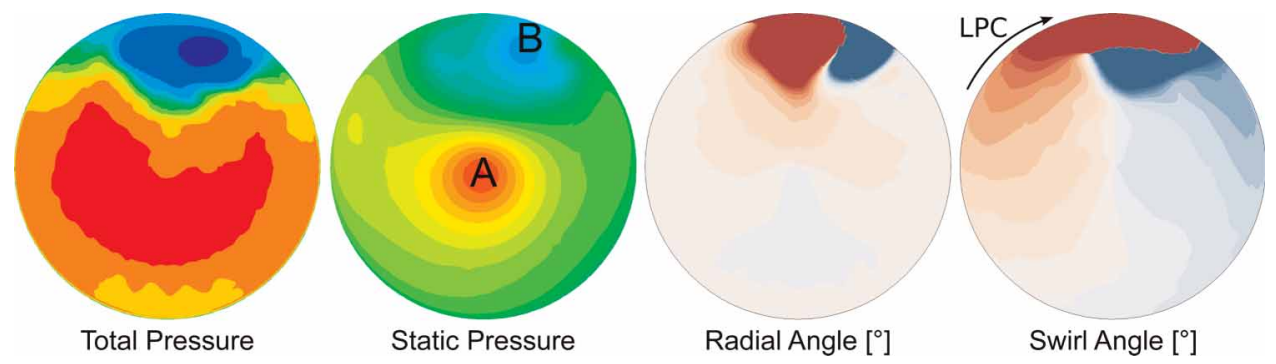

Figure 9. Flow field in cs-6 for combined total pressure swirl distortion (case 3) contour levels according to Figure 5. 
computations. This results in a significant increase computational effort of more than two weeks on 384 CPU. An unsteady OP with variations in $m_{\text {red }}$ of $1.2 \%$ and in $\Pi_{t}$ of about $1 \%$ on periods between 2 and 3 rotor revolutions is observed. The time actual OP thus moves within the orange ellipsis added to Figure 6. For the following comparisons with experimental data, the temporal average over ten rotor revolutions is calculated and analyzed. Again, the mass flow rate is adjusted by matching the $c s-1$ static pressure level, leading to a reduced $p_{\text {out }}$ compared to the cases in the previous section. Due to deviations in the intake loss prediction (cf. Figure 5) also a changed $p_{t, \mathrm{AIP}}$ arises resulting in a reduction of the compressor $p_{t, \mathrm{AIP}} / p_{\text {out }}$ by about $2 \%$. In order to compare the compressor map operation point, these two facts have to be taken into account.

\section{Comparison to experimental data}

Within the first, contracting part of the intake upstream of $c s-3$, a similar pressure distribution is observed between both simulation approaches. After $c s-3$, however, the coupled simulation shows a reduced static pressure along $\phi=0^{\circ}$ compared to the isolated approach, which is caused by the compressor induced static pressure field (cf. Figure 5). In $c s-4$ (cf. Figure 4), the wall pressure distribution of the coupled simulation reveals a slight asymmetry on the upper side, where the local maximum is shifted in positive $y$-direction. This asymmetry is further intensified towards $c s-5$. To visualize the complex character of the flow field in this region, wall streamlines on the upper duct surface for both simulations are presented in Figure 10. Both streamline patterns show a region of backflow downstream of $c s-4$ indicating the occurring flow separation. Within this region, the wall streamlines converge to a spiral node (denoted with $\mathrm{SN}$ ) which is the set off location of a tornado like vertical structure within the flow field. A detailed analysis of this phenomenon can be found in (Wellborn et al., 1993). In case of the coupled approach (cf. Figure 10b), this spiral node is clearly shifted in positive $y$-direction.

Towards the AIP, the induced static pressure field within the coupled simulation leads to a reproduction of two measured pressure characteristics, which the isolated intake simulation was unable to predict: The local pressure minimum at $\phi=0^{\circ}$ for $x / D_{\mathrm{AIP}}>-0.22$ (in experiments at $x / D_{\mathrm{AIP}}>-0.28$ ) and the subsequent pressure rise towards the AIP. Even though such a pressure rise after a pressure plateau is often referred to a reattachment of the flow, backflow is clearly visible in the wall streamline plots as well as in the AIP (cf. Figure 5). Compared to the isolated intake simulations, however, the extent of the region of backflow is reduced, which leads to an improved prediction of the radial and circumferential flow angles. Again, the total pressure distortion of the intake is under predicted by CFD. The LPC in the coupled simulations thus experiences a reduced total pressure distortion resulting in a weaker induced static pressure field compared to the experiment.

\section{Asymmetric AIP flow field}

Again, a slight phase shift between the center of the total pressure distortion and the center of the induced static pressure distortion in clockwise (positive rotor rotation) direction is present as it was already seen in cases 2 and 3. It can therefore be identified as a feature of the numerical modeling of the LPC, as it was not measured to that extent in experiments. The asymmetry of the AIP flow field in the coupled simulations is thus composed of two isolated phenomena: First is the already slight asymmetry of the isolated intake flow field (cf. Figure 5) due to inhomogeneous intake inflow. Second is the shift of the induced static pressure distortion in co-rotating direction by the LPC simulation. Both phenomena superpose in case of the coupled simulations and lead to a strong shift of the static pressure distortion, the region of flow separation and the resulting total pressure distortion in circumferential direction.

As the CFD intake flow field shows an increasing asymmetry between $c s-3$ and AIP in clockwise direction, a further movement of the total pressure distortion is expected towards the rotor inlet plane. The AIP flow field
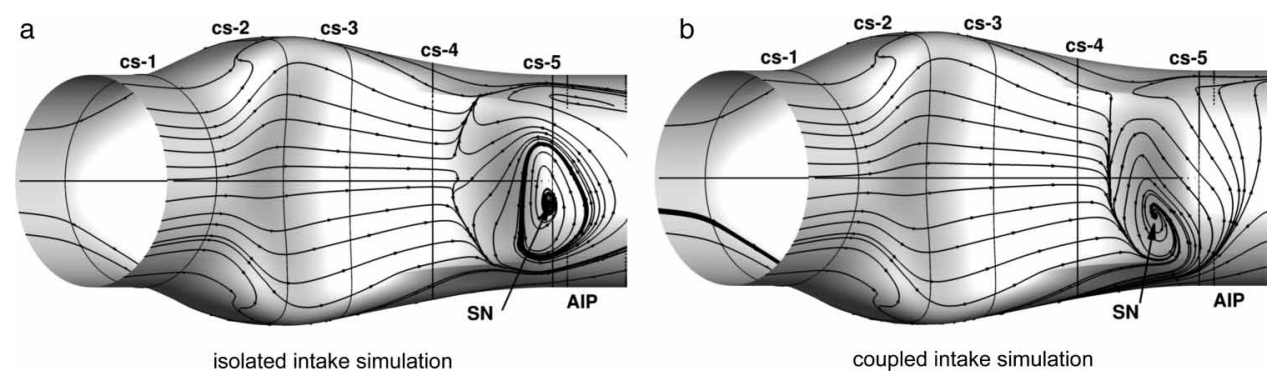

Figure 10. Top view on intake with wall streamlines. 
thus shows already a decoupling of the pressure distortions leading to a circumferential extension and thus mitigation of the total pressure distortion.

\section{Conclusions}

The flow field in a coupled intake-compressor system was investigated by means of numerical flow simulations. For validation purposes, test data from an extensive measurement campaign of a full size system was available. Two characteristics were of particular interest: The precise prediction of the AIP flow distortion and the intake-compressor interaction. Three simulation approaches were investigated:

1. An isolated intake simulation requires minor computational resources and calculation time to deliver a converged solution. It is able to reproduce the intake wall pressure field and predicts the occurring flow separation. In case of the investigated duct geometry, however, the AIP lies very close to the separation location. Without the compressor induced static pressure field, the AIP flow distortion predicted by this approach shows backflow and lacks in precision, especially regarding the swirl distortion. The calculated AIP total pressure distortion is stretched in circumferential extent with the minimum total pressure values are underestimated by approximately 5\%. Using a two dimensional static pressure outlet boundary condition, the prediction of the flow angles can be improved, but the total pressure distortion remains significantly under predicted.

2. The influence of the specific inflow distortion components on the operational behavior of the LPC was assessed by means of isolated compressor simulations. An increase in mass flow rate was observed for the investigated swirl distortion, which is not yet fully understood. The influence of the total pressure component of the distortion is comparable to a respective throttling of the compressor and moves the operational point along the speed line but with a further reduction in $m_{\text {red }}$ due to an induced swirl distortion. The shift in operation point through the combined pressure swirl distortion is mainly a superposition of the two previous cases. The induced static pressure field as a reaction of the compressor on the total pressure distortion was under predicted compared to measured data.

3. Finding the respective operation point for the coupled calculation of intake and compressor is challenging: An adjustment according to the measured intake inflow Mach number leads to a reduced compressor $m_{\text {red }}$ due to an under prediction of the intake total pressure losses by the used numerical setup. In addition with the previously mentioned under prediction of induced static pressure field, the computed interaction between intake and compressor is weaker compared to measurement data. While the engine showed a steady operation point during experiments, computations revealed an unsteady operation point varying by approximately $1 \%$ in $m_{\text {red }}$ and $\Pi_{t}$.

The under prediction of the s-duct total pressure distortions is a general problem of RANS computations. The special design of the investigated intake geometry, however, with a severe flow separation shortly upstream of the AIP is a very challenging test case for a proper prediction of the AIP flow angles. Using coupled intakecompressor simulations only leads to a slight improvement, as the upstream static pressure field is under predicted by CFD on the one hand and dependent on the incoming total pressure distortion on the other hand. Further investigations will focus on the reasons of the observed unsteadiness of the operation point as well as the asymmetry of the intake flow field. Therefore, additional operation points are simulated. Additionally, the distortion transport through the three stages of the compressor and the decoupling of the specific distortion patterns is of interest.

\section{Nomenclature}

\section{Symbols}

A cross-sectional area

$\alpha_{R} \quad$ radial flow angle

$D$ diameter

$\Delta$ difference

$\phi \quad$ circumferential coordinate

$m_{\text {red }}$ mass flow parameter

$p$ pressure

$\Pi_{t} \quad$ total pressure ratio 


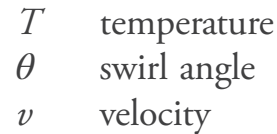

\title{
Subscripts
}

$R$ radial

rel relative

$t$ total

$\theta$ circumferential

\section{Abbreviations}

\author{
AIP Aerodynamic Interface Plane \\ BLI Boundary Layer Ingestion \\ CPU Central Processing Unit \\ Cs cross section \\ DIP Duct Inlet Plane \\ EXP Experimental \\ LPC Low Pressure Compressor \\ Nlc corrected low pressure spool speed \\ Norm normalized value \\ $\mathrm{OP}(\mathrm{s})$ Operation Point(s) \\ SN Spiral Node
}

\section{Acknowledgements}

The presented simulations were carried out within the scope of the COORETEC-Turbo 2020 project no. 1.2.4a. Stabilität des Verdichtersystems bei Off-Design Zuströmbedingungen. This AG Turbo project is funded by the German Ministry of Economy and Technology (BMWi) and conducted in cooperation with MTU Aero Engines AG.

\section{Competing interests}

Thomas Kächele declares that he has no conflict of interest. Rudolf P.M. Rademakers declares that he has no conflict of interest. Tim Schneider declares that he has no conflict of interest. Reinhard Niehuis declares that he has no conflict of interest.

\section{References}

Cumpsty N. A. (2010). Some lessons learned. Journal of Turbomachinery. 132 (4): 041018. https://doi.org/10.1115/1.4001222

Delot A.-L. and Scharnhorst R. K. (2013). A Comparison of Several CFD Codes with Experimental Data in a Diffusing S-Duct. In: Proceedings of 49th AIAA/ASME/SAE/ASEE Joint Propulsion Conference. https://doi.org/10.2514/6.2013-3796

Gunn E. J. and Hall C. A. (2014). Aerodynamics of boundary layer ingesting fans. In: Proceedings of ASME Turbo Expo 2014: Turbine Technical Conference and Exposition. https://doi.org/10.1115/GT2014-26142

Gunn E. J., Tooze S. E., Hall C. A., and Colin Y. (2013). An experimental study of loss sources in a fan operating with continuous inlet stagnation pressure distortion. Journal of Turbomachinery. 135 (5): 051002. https://doi.org/10.1115/1.4007835.

Kächele T., Niehuis R., and Schneider T. (2016). Study on efficient full annulus URANS computations of an intake compressor configuration. In: Proceedings of 30th Congress of the International Council of the Aeronautical Sciences, Daejeon, Korea. ICAS2016-0548.

Kächele T., Schneider T., and Niehuis R. (2018). Steady and Unsteady Numerical Simulation of a Bent Intake Geometry. In: New Results in Numerical and Experimental Fluid Mechanics XI.

Kožulović D. and Röber T. (2006). Modelling the streamline curvature effects in turbomachinery flows. In: ASME Turbo Expo 2006: Power for Land, Sea, and Air. American Society of Mechanical Engineers. https://doi.org/10.1115/GT2006-90265.

Kožulović D., Röber T., Kügeler E., and Nürnberger D. (2004). Modifications of a two-equation turbulence model for turbomachinery fluid flows. In: Deutscher Luft- und Raumfahrtkongress, Dresden, Germany.

Kügeler E. (2005). Numerisches Verfahren zur genauen Analyse der Kühleffektivität filmgekühlter Turbinenschaufeln. DLR Forschungsbericht 2005-11. PhD thesis, Ruhr-Universität Bochum.

Lesser A. and Niehuis R. (2014). Transonic axial compressors with total pressure inlet flow field distortions. In: Proceedings of ASME Turbo Expo 2014: Turbine Technical Conference and Exposition. https://doi.org/10.1115/GT2014-26627

Longley J. P. and Greitzer E. M. (1992). Inlet distortion effects in aircraft propulsion system integration. In: AGARD, Steady and Transient Performance Prediction of Gas Turbine Engines. 
Ma Y., Cui J., Vadlamani N., and Tucker P. (2018). Effect of Fan on Inlet Distortion: A Mixed-fidelity Approach. AIAA Journal. 56 (6): 2350-2360. https://doi.org/10.2514/1.J056858

Mazzawy R. S. (1977). Multiple segment parallel compressor model for circumferential flow distortion. Journal of Engineering for Power. 99 (2): 288-296. https://doi.org/10.1115/1.3446288

Muth B., Opterwinkel N., Bindl S., and Niehuis R. (2009). Numerical Investigation of the Aerodynamic Conditions inside a Sea Level Jet Engine Test Facility Operating a Modern Jet Engine. In: Proceedings of the 19th International Symposium on Air Breathing Engines, Montréal, Canada. ISABE-2009-1214.

Nürnberger D. (2004). Implizite Zeitintregation für die Simulation von Turbomaschinenströmungen. DLR Forschungsbericht $2004-27$. PhD thesis, Ruhr-Universität Bochum.

Page J. H., Hield P., and Tucker P. G. (2017). Effect of Inlet Distortion Features on Transonic Fan Rotor Stall. In: ASME Turbo Expo 2017: Turbomachinery Technical Conference and Exposition. American Society of Mechanical Engineers. https://doi.org/10.1115/ GT2017-64612

Rademakers R. P. M., Kächele T., and Niehuis R. (2016). Integration of a Highly Bent Engine Inlet in an Engine Test Facility. In: Proceedings the International Symposium on Transport Phenomena and Dynamics of Rotating Machinery, Honululu, USA.

Rademakers R. P. M., Probst H., Schneider T., and Niehuis R. (2018). Experimental Investigations on a Bent Engine Inlet Duct Coupled with a Turbofan Engine. In: 2018 AIAA Aerospace Sciences Meeting, Kissimmee, USA. https://doi.org/10.2514/ $6.2018-1353$

Schoenweitz D. and Schnell R. (2016). Development and Evaluation of a Performance Estimation Methodology for Fans Operating Within Non-Homogeneous Inflow. In: ASME Turbo Expo 2016: Turbomachinery Technical Conference and Exposition. GT2016-57095. American Society of Mechanical Engineers. https://doi.org/10.1115/GT2016-57095

Sheoran Y., Bouldin B., and Krishnan P. M. (2012). Compressor performance and operability in swirl distortion. Journal of Turbomachinery. 134 (4): 041008. https://doi.org/10.1115/1.4003657

Tormalm M. (2006). Design and Analysis of Compact UAV Ducts. In: 24th Applied Aerodynamics Conference. https://doi.org/10.2514/ $6.2006-2828$

Wellborn S. R., Okiishi T. H. and Reichert B. A. (1993). A study of the compressible flow through a diffusing S-Duct. Tech. rep. NASA-TM-106411. NASA Lewis Research Center.

Weston D. B., Gorrell S. E., Marshall M. L. and Wallis C. V. (2015). Analysis of turbofan performance under total pressure distortion at various operating points. In: Proceedings of ASME Turbo Expo 2015: Turbine Technical Conference and Exposition. Vol. 34. GT2015-42879. https://doi.org/10.1115/GT2015-42879

Yao J., Gorrell S. E., and Wadia A. R. (2010a). High-fidelity numerical analysis of per-rev-type inlet distortion transfer in multistage fans —Part I: Simulations with selected blade rows. Journal of Turbomachinery. 132 (4): 041014. https://doi.org/10.1115/1.3148478

Yao J., Gorrell S. E., and Wadia A. R. (2010b). High-fidelity numerical analysis of per-rev-type inlet distortion transfer in multistage fans —Part II: Entire component simulation and investigation. Journal of Turbomachinery. 132 (4): 041015. https://doi.org/10. 1115/ 1.3148479 